\title{
ALCity: Uma Ferramenta de Autoria para o Desenvolvimento de Jogos Móveis Baseados em Localização para Apoiar o Processo de Ensino
}

\author{
Ivan R. de Moura ${ }^{1}$, Carleandro de O. Nolêto ${ }^{1}$, Igor Feliciano S. Revoredo ${ }^{1}$ \\ ${ }^{1}$ Estácio de Sá - CEUT \\ Coordenação de Ciência da Computação -Teresina,PI - Brasil \\ \{ivanrmoura9, carleandro7, igorrevoredo\}@gmail.com
}

\begin{abstract}
The development of new forms of teaching is challenging and can be considered complex to implement. One of the existing tools that has the potential to meet this need is the location-based mobile games, since it has the characteristics of an entertainment activity, facilitating the learning process. This article conceived an authoring tool titled ALCity (Locative Adventures in the City), which allows teachers to design location-based mobile games with the integration of augmented reality feature to support the teaching and learning process. The usability of the designed authoring tool was evaluated by a set of experienced teachers. From this evaluation, it was observed that ALCity is able to efficiently assist the teaching process.
\end{abstract}

Resumo. O desenvolvimento de novas formas de ensino é algo desafiador, podendo ser considerado complexo de ser implementado. Uma das ferramentas existentes que possui o potencial para suprir tal necessidade é o jogo móvel baseado em localização (JMBL), pois tem como característica ser uma atividade de entretenimento, facilitando assim o processo de aprendizado. Este artigo concebeu uma ferramenta de autoria intitulada ALCity (Locative Adventures in the City), que permite professores desenvolverem JMBLs com a integração do recurso de realidade aumentada, com o propósito de apoiar o processo de ensino e aprendizado. A usabilidade da ferramenta de autoria concebida foi avaliada por um conjunto de professores experientes. A partir desta avaliação, observou-se que a ALCity consegue, de forma eficiente, auxiliar o processo de ensino.

\section{Introdução}

Atualmente, os jogos digitais são uma tecnologia cada vez mais presente em nosso cotidiano, que oferecem uma atividade lúdica capaz de capturar nossa atenção por um longo tempo. Com os avanços de hardware e software dos dispositivos móveis, houve-se a potencialização do seu uso como uma plataforma para jogos digitais, onde possibilitam uma interação com o usuário bastante intuitiva, oferecendo ainda uma serie de sensores capazes de reconhecer o contexto em que o usuário está inserido, como localização, altitude, velocidade, temperatura, etc.

Essas características dos dispositivos móveis, possibilitaram o surgimento de uma classe especial de jogos digitais, denominada Jogos Móveis Baseados em Localização (JMBLs). Estes são uma subclasse dos jogos pervasivos, onde a localização do jogador 
VI Congresso Brasileiro de Informática na Educação (CBIE 2017)

Anais do XXVIII Simpósio Brasileiro de Informática na Educação (SBIE 2017)

deve fazer parte de suas regras e mecânicas. Os jogos pervasivos não se restringem ao espaço virtual das telas dos computadores, extrapolam estes limites, passando a integrar aos aspectos físicos e sociais do mundo real [Magerkurth et al. 2005]. [Lemos 2010] define os JMBLs como jogos que se baseiam na localização do usuário para as tomadas de decisões, criando assim uma interface entre os espaços físicos e virtuais. Eles possibilitam que ações realizadas pelos jogadores no espaço real influencie seu desempenho no espaço virtual, alguns exemplos de JMBLs são: Ingress, que foi desenvolvido pela startup Niantic do Google, Pokemon GO, concebido por meio da colaboração entre Niantic, Nintendo e The Pokémon Company. Deste modo, oferecem aos seus jogadores experiências inovadoras, capazes de atrair a atenção e interesse de um grande número de adeptos. A tecnologia de realidade aumentada (RA) pode ser integrada aos JMBLs, segundo [Kirner and Zorzal 2005] a RA consiste na sobreposição de ambientes reais e virtuais, em tempo real, através de um dispositivo tecnológico, potencializando assim o poder de entretenimento da experiência oferecida pelo JMBL.

As características apresentadas pelos JMBLs torna esse tipo de jogo uma tecnologia em potencial de modo a colaborar no processo de ensino e aprendizado de forma prazerosa. Com isso, existe a possibilidade do professor adicionar em sua didática de ensino a aplicação de JMBLs educativos, conseguindo assim gamificar o processo de ensino. Porém, há um empecilho para que essa metodologia se torne viável, que é o fato do processo de desenvolvimento de um jogo digital não ser trivial, onde é requerido o conhecimento de várias tecnologias que são aplicadas no processo, além de necessitar do conhecimento de especialistas de diferentes áreas, como por exemplo, programadores, designs, sonoplastas, roteiristas, etc, ou seja, é um processo complexo, ainda mais se tratando de um JMBL com RA, que se deve ter a preocupação com contexto em que o usuário está inserido, tornando ainda maior o desafio do desenvolvimento. O professor que não possui o conhecimento necessário para produção, não terá êxito nesta tarefa. Assim, tornar-se necessário soluções que capacite professores a criarem seus jogos, mesmo sem o conhecimento necessário.

O objetivo deste trabalho é gerar uma ferramenta de autoria denominada ALCity (Locative Adventures in the City), para permitir que professores concebam seus próprios JMBLs com a integração de realidade aumenta com objetos em 2D, onde é possível aplicar o conteúdo a ser lecionado. ALCity irá abstrair a necessidade do conhecimento de uma linguagem de programação por parte do utilizador e apresentará para o usuário uma interface intuitiva, ou seja, sem a necessidade de grande esforço para conseguir interagir com a ferramenta concebida.

Este artigo está organizado da seguinte forma: a Seção 2 apresenta a metodologia usada para conceber este artigo; a Seção 3 discute os conceitos sobre jogos móveis baseados em localização; a Seção 4 apresenta a ferramenta de autoria concebida; a Seção 5 expõe uma avaliação realizada sobre a ferramenta de autoria concebida; a Seção 6 apresenta a conclusão e os trabalhos futuros sobre este artigo.

\section{Metodologia da Pesquisa}

Para a produção deste artigo foram realizadas algumas etapas. A etapa de levantamento bibliográfico tipo exploratório foi executada com o objetivo de obter conhecimentos teóricos e técnicos sobre os JMBLs e de sua integração com realidade aumentada. Pos- 
VI Congresso Brasileiro de Informática na Educação (CBIE 2017)

Anais do XXVIII Simpósio Brasileiro de Informática na Educação (SBIE 2017)

teriormente realizou-se a etapa de estudo de trabalhos existentes que possuem relação com este artigo, buscou-se descrever a problematização que o artigo busca sanar, citada anteriormente, assim como a solução proposta. Com base nas informações levantadas, buscou-se o início do processo de desenvolvimento da ferramenta de autoria proposta. Inicialmente realizou-se o levantamento de requisitos e modelagem, por fim executou-se a implementação.

Com objetivo de avaliar a ergonomia de design apresentada pela ferramenta de autoria ALCity, selecionou-se um conjunto de professores pertencentes a áreas distintas de conhecimento, foi incumbido a estes professores a tarefa de criar um JMBL através da ALCity. Após a experiência de uso, foi aplicado a estes professores o formulário ISONORM 9241/110-S [PRÜMPER ], que foi modelado com base nos sete princípios de diálogo apresentados na Norma ISO 9241-110.

\section{Fundamentação Teórica}

Nesta seção será apresentado conceitos importantes para o desenvolvimento do presente trabalho. Serão abordados temas com relação aos jogos móveis baseados em localização no intuito de esclarecer suas características e potenciais.

\subsection{Jogos Móveis Baseados em Localização}

Em um passado próximo o espaço urbano era muito utilizado para atividades lúdicas, onde se exercitava a comunicação e convívio, ou seja, havia interação com o mundo externo, mas com a chegada dos consoles dos jogos digitais, o número de adeptos das atividades urbanas diminuiu, onde houve-se o temor que ocorresse o aprisionamento do jogador as telas dos consoles. Os JMBLs podem quebrar este paradigma, mostrando que os jogos digitais podem extrapolar as telas dos consoles para o espaço urbano, onde o jogador passa a vivenciar um jogo que tem relação com o mundo real e está potencialmente disponível em qualquer lugar e a qualquer hora [Benford et al. 2005]. Nos JMBLs, os jogadores usam o espaço urbano como cenário de jogo, os jogadores portam consigo um dispositivo móvel que possui algum tipo de sensor de localização, onde ela influi no desempenho do jogador.

Os JMBLs são uma subclasse dos jogos pervasivos, que são definidos como jogos que invadem o espaço urbano e a vida cotidiana, criando uma interface entre os espaços virtual e físico [Montola et al. 2009]. Os JMBLs utilizam as mídias locativas para se apropriarem do espaço urbano. As mídias locativas são definidas como um conjunto de tecnologias e processos info-comunicacionais cujo conteúdo informacional vincula-se a um lugar específico [Lemos 2007]. São exemplos de mídias locativas o Sistema de Posição Global(GPS - Global Positioning System), QR Codes, dispositivos móveis, etc. É através delas que se torna possível a implementação da principal característica dos JMBLs, a integração da localização do jogador na sua lógica e regras. A tecnologia de Realidade Aumentada(RA) pode ser integrada aos JMBLs para potencializar suas atividades lúdicas. A ALCity possibilita a criação de JMBLs com realidade aumentada, onde será possível adicionar objetos $2 \mathrm{D}$ sobre o mundo real.

\subsection{Trabalhos Relacionados}

ALRA (Aventuras Locativas em Realidade Aumentada) é uma ferramenta de autoria que possui entre suas características a criação e execução de JMBLs com realidade aumen- 
VI Congresso Brasileiro de Informática na Educação (CBIE 2017)

Anais do XXVIII Simpósio Brasileiro de Informática na Educação (SBIE 2017)

tada. Esta ferramenta possibilita a criação colaborativa de novas aventuras assim como a vivência por meio de um dispositivo móvel ou de desktop [dos Santos et al. 2013]. Ela é composta por quatro ambientes: o primeiro é a página web para a criação dos jogos; segundo é o ambiente de execução dos jogos através de um dispositivo móvel; o terceiro é um ambiente para a criação e configuração dos jogadores; e por último um ambiente de monitoramento e controle das ações dos jogadores.

Outra ferramenta que pode ser citada é a Tidy City Scout, que possibilita seus usuários criarem conteúdos próprios, adicionando missões que envolvem um nível de dificuldade, uma categoria, um texto de pista e uma imagem de indício, criando a partir destas missões enigmas que são inseridos em pontos da cidade. Com a ajuda de seus smartphones, os jogadores pegam enigmas que estão espalhados em torno do ambiente e mostra em um mapa. Esta ferramenta é executada na plataforma android e apresenta uma interatividade fácil para a criação das mecânicas do jogo [Wetzel et al. 2012].

\section{ALCity - Ferramenta de Autoria de Jogos Móveis Baseados em Localização}

\subsection{Arquitetura}

Após o termino do processo de revisão da literatura, foi possível identificar as características dos JMBLs. A partir dessas informações levantadas neste processo, foi elaborado uma arquitetura de software que possibilita gerenciar todo o processo que envolve a ALCity, desde a modelagem, até a execução do JMBL gerado. A arquitetura é composta por três componentes: editor do jogo, servidor e aplicação móvel. O servidor é responsável por gerenciar as informações sobre os jogadores e jogos. A aplicação móvel foi concebida para a plataforma Android, onde a mesma é responsável pela execução dos jogos desenvolvidos no editor, é através deste componente que os alunos participam da atividade lúdica proposta pelos professores. A arquitetura foi construída baseada no modelo cliente-servidor, onde o servidor é o componente central que fornece serviços aos demais componentes. A Figura 1 apresenta uma visão geral da arquitetura desenvolvida.

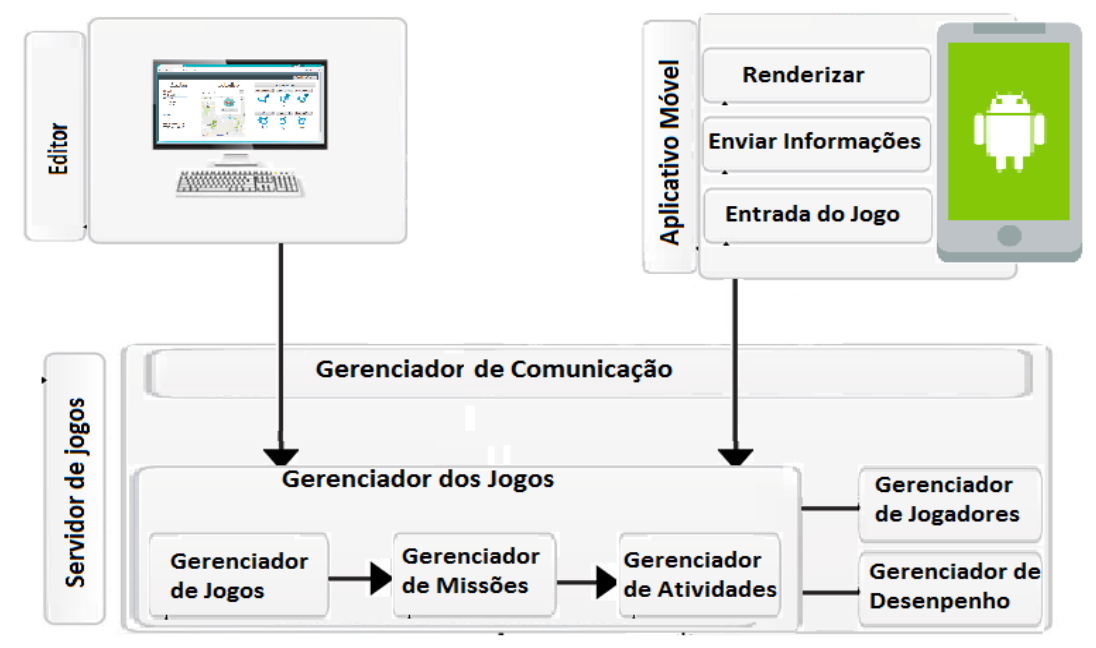

Figura 1. Arquitetura da ALCity 
VI Congresso Brasileiro de Informática na Educação (CBIE 2017)

Anais do XXVIII Simpósio Brasileiro de Informática na Educação (SBIE 2017)

\subsubsection{Servidor}

É o componente responsável por responder a todas solicitações de serviços realizadas pelo editor e aplicação móvel, ou seja, é o componente que capacita os demais a funcionar satisfatoriamente. Este é divido em seis componentes: gerenciador de comunicação, gerenciador de jogos, gerenciador de missões, gerenciador de atividades, gerenciador de jogadores e gerenciador de desempenho.

O gerenciador de comunicação é o componente responsável por controlar o fluxo de informações entre o servidor e os demais softwares que compõem a arquitetura. Todos os dados dos jogos e jogadores são tratados por esse componente.

O gerenciador de jogos é responsável por controlar todos os jogos desenvolvidos pela ALCity. Os jogadores solicitam acesso aos dados dos jogos ao servidor, neste momento, este componente intercepta a requisição, e identifica qual o jogo requerido. Já o gerenciador de jogadores controla informações referentes aos jogadores, como seus jogos, missões, desempenho.

O gerenciador de missões é responsável por controlar todas as missões de cada jogo, desde a sua configuração, até a realização. Este componente também é responsável por gerenciar os estados das missões, permitindo assim a identificação do progresso de cada jogador. Cada missão possui um tipo de atividade lúdica, podendo ser ela, responder um quiz locativo ou capturar um objeto com realidade aumentada. O controle destas atividades é responsabilidade do gerenciador de atividades.

O gerenciador de desempenho controla os dados estatísticos dos jogadores em cada jogo. É através deste componente que se torna possível montar relatórios sobre as atividades lúdicas praticadas pelos jogadores.

\subsubsection{Editor do Jogo}

É uma aplicação web que possibilita professores a modelar e conceber JMBLs. Sua interface deve conseguir se comunicar com o usuário de forma que não necessite grande esforço para se alcançar exito nesta interatividade entre software e usuário. Para suprir esta necessidade, foi realizado um estudo dos trabalhos de [Wang et al. 2011] e [Bichard et al. 2006]. Estes autores apresentam em seus trabalhos, uma interface que permite usuários não programadores a desenvolverem seus JMBLs. A ferramenta concebida por [Wang et al. 2011] se utiliza de blocos para criar as regras e mecânicas dos jogos. Para conceber sua ferramenta, [Bichard et al. 2006] realizou um estudo das interfaces de outras ferramentas de autorias e efetuou entrevistas com desenvolvedores experientes de um JMBL. As duas ferramentas mencionadas possuem características semelhantes em suas interfaces, o mapa e as mecânicas do jogo ficam posicionado sempre ao centro da interface, com as paletas que são usadas para adicionar as mecânicas ao jogo ficam dispostas em suas laterais. Por meio dos resultados destes trabalhos foi possível desenvolver o designer da interface do editor web.

Os professores poderão acessar o editor do jogo de qualquer dispositivo digital, com um navegador web e acesso a internet. O professor deverá criar um perfil na tela de cadastro, liberando assim a utilização de todas as funcionalidades oferecidas pelo editor. 
VI Congresso Brasileiro de Informática na Educação (CBIE 2017)

Anais do XXVIII Simpósio Brasileiro de Informática na Educação (SBIE 2017)

A tela de modelagem do jogo (Figura 2) é onde se localiza a maioria das configurações, possui um mapa com a localização e descrição de todas missões e atividades já criadas, deste modo, é possível identificar visualmente toda a estrutura do jogo que está em desenvolvimento. A partir desta é feita a navegação para a tela de modelagem das missões, onde oferece a funcionalidade de criar atividades, que são adicionadas a lista de atividades da missão em desenvolvimento.

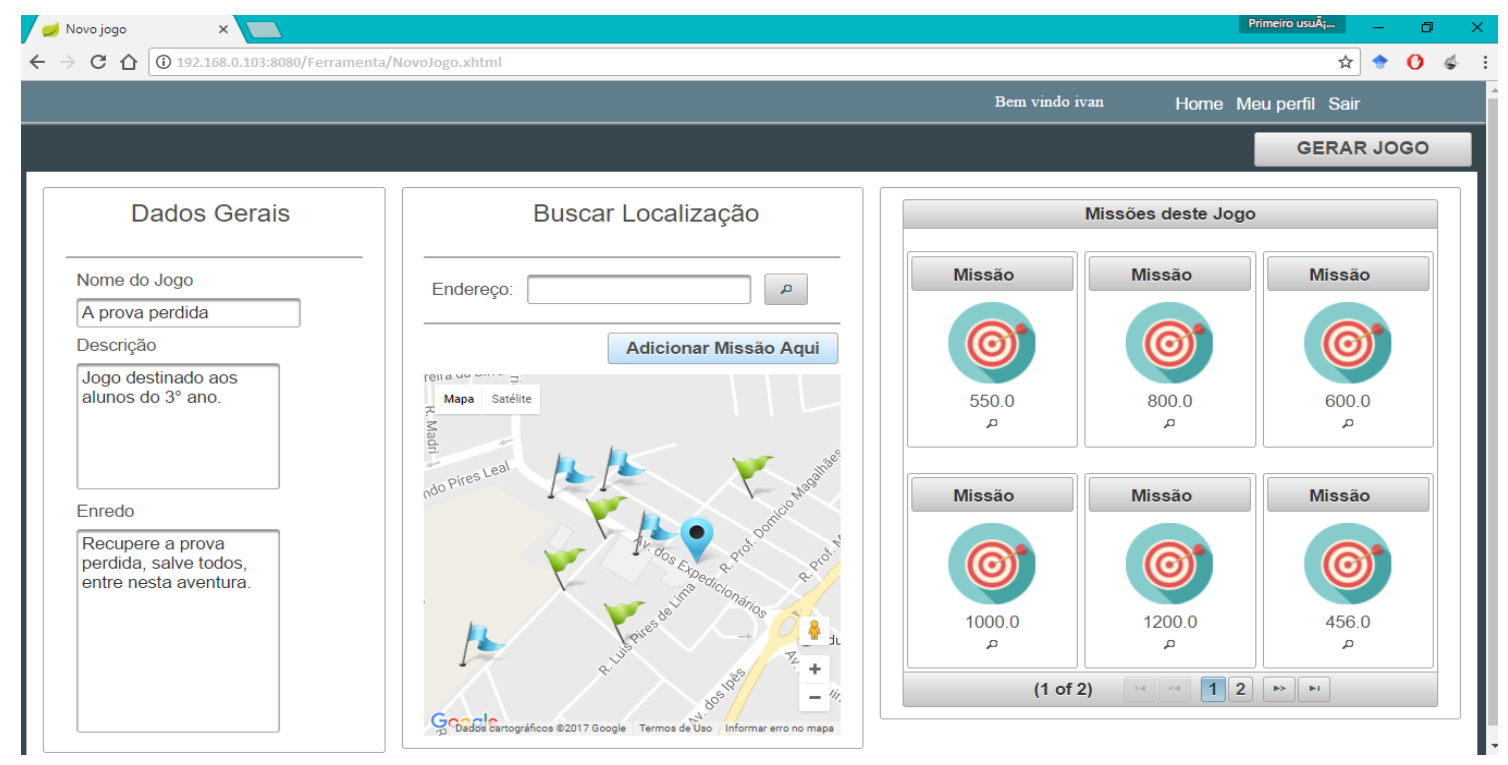

Figura 2. Interface para modelagem de um novo jogo

As atividades disponíveis são: quiz locativo e capturar um objeto de realidade aumentada. O quiz locativo necessita que o aluno se locomova até um ponto do espaço urbano, onde responderá um quiz, obtendo assim uma pontuação. $\mathrm{Na}$ atividade capturar um objeto de realidade aumentada, necessita que o aluno se locomova até um ponto do espaço urbano, identifique e capture um objeto, recebendo logo após a conclusão, uma mensagem. Todas as atividades são desenvolvidas de forma que insira o aluno dentro de um ambiente lúdico, com objetivo de engajar o aluno na atividade proposta pelo professor.

\subsubsection{Aplicação Móvel}

É o componente responsável por oferecer o ambiente lúdico modelado pelos professores no editor, interage diretamente com os alunos. Foi desenvolvido para ser suportado pela plataforma android. Sua interface foi projetada para operar de forma responsiva, ou seja, capaz de se adaptar a todos tamanhos de telas dos dispositivos móveis, objetivando oferecer uma melhor experiência de uso.

Para ter acesso a todas as funcionalidades disponíveis, o aluno deve criar um perfil na tela de cadastro, logo após estará apto a participar das atividades lúdicas. Cada jogo modelado pelo editor possui um código identificador, os professores são responsáveis por divulgar este código aos seus alunos alvos, ou seja, a quem interessa a atividade proposta. Com posse deste, os alunos podem realizar o download dos arquivos necessários para a execução do JMBL criado, de forma rápida e fácil, ao termino desta tarefa, o aluno se tornará um competidor, onde suas ações dentro do jogo afeta seu desempenho. 
VI Congresso Brasileiro de Informática na Educação (CBIE 2017)

Anais do XXVIII Simpósio Brasileiro de Informática na Educação (SBIE 2017)

A interface do JMBL em execução (Figura 3) é bem intuitiva, ela é basicamente composta por dois elementos: um mapa com as localizações das missões disponíveis (3(a)) e uma aba que dá acesso a mochila contendo todos os objetos capturados pelo jogador. $\mathrm{O}$ aluno pode executar dois tipos de atividades lúdicas propostas pelos educadores, capturar um objeto de realidade aumentada (Figura 3(b)) e responder um quiz locativo (Figura 3(c)).

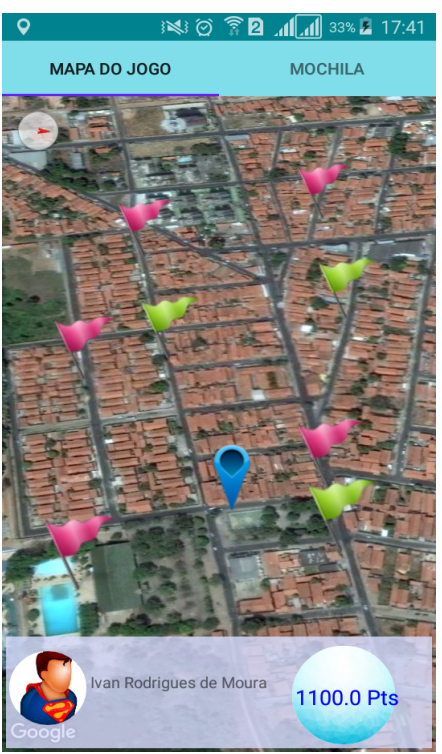

(a) Tela Principal com as missões

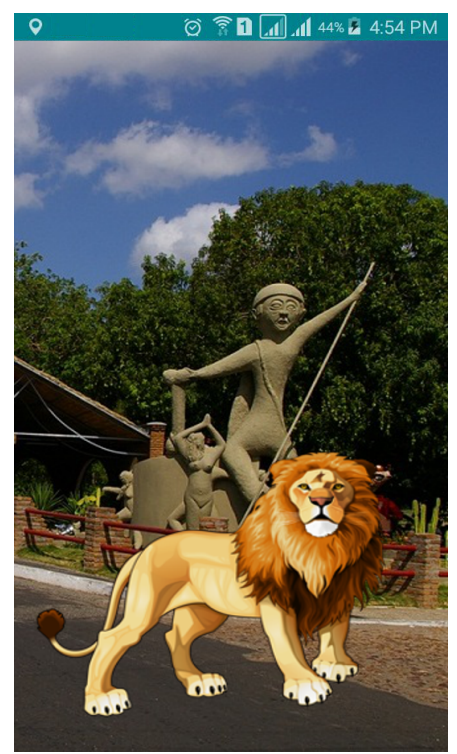

(b) Missão Capturar Objeto de RA

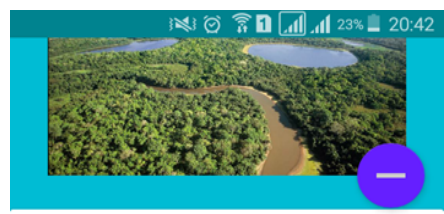

Questão: Quiz 01

Questão: sou o maior felino do continente americano. peso entre 35 e $130 \mathrm{~kg}$. Tenho o corpo robusto, compacto e musculoso. Minha pelagem varia do amarelo-claro ao castanho-ocreáceo e é caracterizada por manchas pretas em forma de rosetas de diferentes tamanhos.

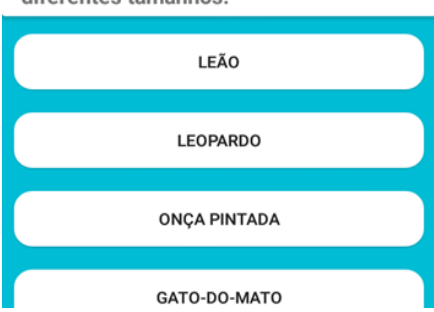

(c) Missão Quiz Locativo

\section{Figura 3. Telas do aplicativo móvel}

\section{Avaliação}

A usabilidade avalia a simplicidade e eficiência da interface de usuário. Assim, um bom software deve possibilitar seus usuários utiliza-lo da melhor maneira possível, permitindo executar suas tarefas sem dificuldades, hesitação ou dúvidas. Para avaliar a usabilidade da ALCity, foi aplicado o formulário ISONORM 9241/110-S [PRÜMPER ], que foi modelado baseado nos sete princípios de diálogo apresentados na Norma ISO 9241-110.

\subsection{Norma ISO 9241-110}

A ISO 9241-110: 2006 determina princípios referentes a ergonomia de design para sistemas interativos. Esta norma é dividida em sete princípios de diálogo formulados em termos gerais, ou seja, retratados sem referência a situações de uso, aplicação, ambiente ou tecnologia. A ISO 9241-110: 2006 centra-se nos princípios de diálogos que possuem relação com a concepção ergonômica do diálogo entre usuário e o sistema, desconsiderando qualquer outro aspecto do design [ISO 2006]. Os princípios são: adequação a tarefa, autodescrição, conformidade com as expectativas do usuário, adequação ao aprendizado, controlabilidade, tolerância a erro e suporte a individualização.

O formulário ISONORM 9241/110-S [PRÜMPER ] individualiza cada princípio da norma ISO 9241-110, onde são medidos a partir de três itens. As respostas são gradativas, se situando entre dois polos, onde o esquerdo é o extremo negativo, e o direito, positivo. A Figura 4 mostra três itens do princípio da tolerância a erros. 
VI Congresso Brasileiro de Informática na Educação (CBIE 2017)

Anais do XXVIII Simpósio Brasileiro de Informática na Educação (SBIE 2017)

\begin{tabular}{|c|c|c|c|c|c|c|c|c|}
\hline & O Software ... & $\ldots$. & . & $.1+$ & + & +. & +++ & O Software ... \\
\hline SW17 & $\begin{array}{l}\text { corrẹāo de erros geralmente } \\
\text { requer um grande esforço. }\end{array}$ & 00 & 0 & 0 & 0 & 0 & 0 & $\begin{array}{l}\text { correçāo de erros geralmente } \\
\text { requer pouco esforço. }\end{array}$ \\
\hline SW18 & $\begin{array}{l}\text { näo fornece uma ajuda } \\
\text { concreta para a corref̧ão de } \\
\text { erros. }\end{array}$ & 00 & 0 & 0 & 0 & 0 & 0 & $\begin{array}{l}\text { formece uma ajuda concreta } \\
\text { para a corrę̧äo de erros. }\end{array}$ \\
\hline SW19 & $\begin{array}{l}\text { é dificil de generalizar se } \\
\text { novas tarefas surgirem para } \\
\text { mim. }\end{array}$ & 00 & 0 & 0 & 0 & 0 & 0 & $\begin{array}{l}\text { Efácil de generalizar se novas } \\
\text { tarefas surgirem para mim. }\end{array}$ \\
\hline
\end{tabular}

Figura 4. Itens do Formulário ISONORM 9241/110-S Tolerância a erros

Esses princípios aplicados ao processo de desenvolvimento da interface de usuário possibilita uma interação humano-computador mais eficiente, evitando problemas de usabilidades, como recuperação de erros ineficientes, informações incompletas, ações desnecessárias para a conclusão de uma tarefa, entre outros.

\subsection{Perfil dos Avaliadores}

Para avaliar a ferramenta de autoria ALCity foi realizada duas etapas. A primeira etapa consistiu em apresentar um projeto de um JMBL educativo para um conjunto de professores com diferentes perfis e áreas de conhecimento. O projeto apresenta um roteiro para o desenvolvimento de um conjunto de missões pré-definidas. Os professores receberam a tarefa de desenvolver o projeto proposto por meio da ALCity. A segunda etapa foi realizada logo após os professores terem acesso a experiência de uso oferecida pela ferramenta, efetuando a aplicação do formulário ISONORM 9241/110-S. Dentro do conjunto de professores que participaram da avaliação, três são doutorando e quatro são graduados, suas áreas de atuação são referentes a telecomunicação, matemática, português, computação, biologia e geografia. Realizou-se um questionário para identificar o perfil dos avaliadores, onde buscou-se elicitar qual o nível de conhecimento técnico os avaliadores possuíam para a produção de um jogo digital. Por meio deste questionário identificou-se que 71 por cento dos avaliadores indicaram não possuir nenhum conhecimento necessário para conceber um jogo digital e que apenas 29 por cento indicaram possuir.

\subsection{Resultados}

Após a aplicação do formulário ISONORM 9241/110-S aos professores selecionados, foi possível identificar as características de usabilidade da ALCity, ou seja, gerou-se uma avaliação da eficiência da interação humano-computador. Os resultados foram tabulados e apresentados na Tabela 1. O resultado foi agrupado segundo os 7 princípios da norma ISO 9241-110.

Observando o princípio da adequação para a tarefa (Prin.1), a ALCity recebeu uma avaliação positiva (soma das colunas,,++++++ ) de $95,29 \%$ e 4,76\% dos avaliadores não indicaram contentamento ou descontentamento (coluna +/-). Autodescrição (Prin.2) recebeu avaliação positiva de $76,25 \%$ e $23,83 \%$ dos avaliadores permaneceram neutro em relação a este princípio. Em relação ao princípio da conformidade com a expectativa do usuário (Prin.3), 100\% dos avaliadores indicaram que a ferramenta satisfaz esse princípio. 
VI Congresso Brasileiro de Informática na Educação (CBIE 2017)

Anais do XXVIII Simpósio Brasileiro de Informática na Educação (SBIE 2017)

Tabela 1. Resultado da aplicação do formulário ISONORM 9241/110-S

\begin{tabular}{l|l|l|l|l|l|l|l}
\hline & --- & -- & - & $+/-$ & + & ++ & +++ \\
\hline \hline Prin.1 & $0 \%$ & $0 \%$ & $0 \%$ & $4,76 \%$ & $23,83 \%$ & $28,6 \%$ & $42,86 \%$ \\
Prin.2 & $0 \%$ & $0 \%$ & $0 \%$ & $23,83 \%$ & $14,3 \%$ & $28,6 \%$ & $33,35 \%$ \\
Prin.3 & $0 \%$ & $0 \%$ & $0 \%$ & $0 \%$ & $19,06 \%$ & $33,35 \%$ & $47,63 \%$ \\
Prin.4 & $0 \%$ & $0 \%$ & $4,76 \%$ & $14,3 \%$ & $0 \%$ & $14,3 \%$ & $66,66 \%$ \\
Prin.5 & $0 \%$ & $4,76 \%$ & $4,76 \%$ & $4,76 \%$ & $19,06 \%$ & $33,33 \%$ & $33,35 \%$ \\
Prin.6 & $0 \%$ & $0 \%$ & $0 \%$ & $0 \%$ & $0 \%$ & $29,4 \%$ & $70,6 \%$ \\
Prin.7 & $0 \%$ & $0 \%$ & $0 \%$ & $9,54 \%$ & $19,06 \%$ & $9,54 \%$ & $61,86 \%$ \\
\hline
\end{tabular}

O princípio adequação (Prin.4) para o aprendizado alcançou avaliação positiva de $80,79 \%, 14,13 \%$ permaneceram neutro e o percentual de avaliações negativas (soma das colunas -, - -, - - -) alcançou 4,76\%. Uma pequena parte teve dificuldade no aprendizado oferecido pela ALCity, citando que a mesma exige memorização de detalhes.

Segundo o princípio da controlabilidade (Prin.5), 85,74\% dos avaliadores indicaram que a ALCity atende a este princípio, 4,76\% permaneceram neutros e 9,52\% indicaram índices negativos. Este foi o pior princípio avaliado, identificando que a ALCity força o usuário a seguir uma sequência de passos desnecessariamente rígida.

Em relação ao princípio de tolerância a erro (Prin.6), 100\% dos avaliadores demostraram está satisfeito com a usabilidade oferecida pela ferramenta. Este foi o princípio que recebeu a maior avaliação positiva.

Suporte para individualização (Prin.7) recebeu avaliação positiva de 90,46\%, 9,54\% não demostraram contentamento ou descontentamento em relação a este princípio.

A partir dos resultados obtidos através da execução desta avaliação, foi possível identificar que a ALCity satisfaz a maioria dos princípios de usabilidade avaliados, permitindo ainda, a identificação das características que devem ser melhoradas na usabilidade da ferramenta concebida, como a controlabilidade e adequaçao ao aprendizado.

\section{Conclusão}

Este artigo propôs e concebeu uma ferramenta de autoria que habilita professores, mesmo sem possuir conhecimentos de programação, a desenvolver jogos móveis baseados em localização para auxiliar na sua atividade pedagógica, possibilitando ainda um acompanhamento do desenvolvimento de seus alunos. A ferramenta de autoria cognominada de ALCity oferece um editor web que viabiliza a modelagem e criação de JMBLs, de forma intuitiva e agradável. Os jogos gerados pelo editor são executados a partir de um aplicativo móvel desenvolvido para a plataforma android, nele é possível executar todos os jogos desenvolvidos no editor, ou seja, é o ambiente onde ocorre as atividades lúdicas proposta pelos professores.

A ALCity foi avaliada por professores, através da experiência de uso, logo após, realizou-se a aplicação do formulário ISONORM 9241/110-S, onde obteve-se resultados bastante satisfatórios, em especial os princípios adequação para a tarefa, conformidade com a expectativa do usuário, tolerância a erros e Adequação para individualização, que receberam avaliações superiores aos noventa por cento. Através desta avaliação foi identificados os pontos a serem melhorados na ferramenta, que serão implementados em um 
VI Congresso Brasileiro de Informática na Educação (CBIE 2017)

Anais do XXVIII Simpósio Brasileiro de Informática na Educação (SBIE 2017)

trabalho futuro.

Como trabalho futuro pretende-se acoplar na ALCity a opção de utilizar objetos multimídias, como vídeos, áudios e adicionar a possibilidade de utilizar objetos 3D. Pretende-se ainda realizar avaliações da usabilidade dos jogos gerados pelo editor.

\section{Referências}

Benford, S., Magerkurth, C., and Ljungstrand, P. (2005). Bridging the physical and digital in pervasive gaming. Communications of the ACM, 48(3):54-57.

Bichard, J., Brunnberg, L., Combetto, M., Gustafsson, A., and Juhlin, O. (2006). Backseat playgrounds: pervasive storytelling in vast location based games. In International Conference on Entertainment Computing, pages 117-122. Springer.

dos Santos, O. L., do Prado Rafalski, J., and de Menezes, C. S. (2013). Uma game engine para aventuras pedagógicas locativas em realidade aumentada. In Brazilian Symposium on Computers in Education (Simpósio Brasileiro de Informática na Educação-SBIE), volume 24, page 396.

ISO (2006). ISO 9241-110: Ergonomics of Human-system Interaction-Pt. 110: Dialogue Principles. ISO.

Kirner, C. and Zorzal, E. R. (2005). Aplicações educacionais em ambientes colaborativos com realidade aumentada. In Brazilian Symposium on Computers in Education (Simpósio Brasileiro de Informática na Educação-SBIE), volume 1, pages 114-124.

Lemos, A. (2007). Mídia locativa e territórios informacionais. Information media.

Lemos, A. (2010). Jogos móveis locativos: Cibercultura, espaço urbano e mídia locativa. Revista USP, (86):54-65.

Magerkurth, C., Cheok, A. D., Mandryk, R. L., and Nilsen, T. (2005). Pervasive games: bringing computer entertainment back to the real world. Computers in Entertainment (CIE), 3(3):4-4.

Montola, M., Stenros, J., and Waern, A. (2009). Pervasive games: theory and design. Morgan Kaufmann Publishers Inc.

PRÜMPER, J. Isonorm 9241/110-s: Beurteilung von software auf grundlage der internationalen ergonomie-norm din en iso 9241-110. Access date: 18 fev. 2017.

Wang, A. I., Jurgelionis, A., Guo, H., and Trætteberg, H. (2011). Designing enhanced authoring tools for pervasive games. In Mobile Gaming workshop (moga) 2011 on the 8th International Conference on Advances in Computer Entertainment Technology (ACE 2011).

Wetzel, R., Blum, L., and Oppermann, L. (2012). Tidy city: a location-based game supported by in-situ and web-based authoring tools to enable user-created content. In Proceedings of the international conference on the foundations of digital games, pages 238-241. ACM. 